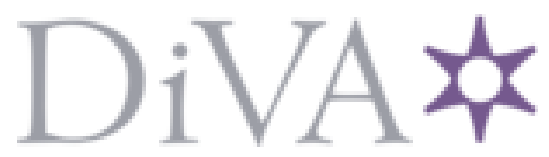

http://www.diva-portal.org

This is the published version of a paper published in Journal of the International Neuropsychological Society.

Citation for the original published paper (version of record):

Stanciu, I., Larsson, M., Nordin, S., Adolfsson, R., Nilsson, L. et al. (2014)

Olfactory Impairment and Subjective Olfactory Complaints Independently Predict Conversion to Dementia: A Longitudinal, Population-Based Study.

Journal of the International Neuropsychological Society, 20(2): 209-217

http://dx.doi.org/10.1017/S1355617713001409

Access to the published version may require subscription.

N.B. When citing this work, cite the original published paper.

Permanent link to this version:

http://urn.kb.se/resolve?urn=urn:nbn:se:umu:diva-88294 


\title{
Olfactory Impairment and Subjective Olfactory Complaints Independently Predict Conversion to Dementia: A Longitudinal, Population-Based Study
}

\author{
Ingrid Stanciu, ${ }^{1,2}$ Maria Larsson, ${ }^{1,2}$ Steven Nordin,${ }^{3}$ Rolf Adolfsson, ${ }^{4}$ Lars-Göran Nilsson, ${ }^{2}$ AND \\ Jonas K. Olofsson ${ }^{1,2,5}$ \\ ${ }^{1}$ Gösta Ekman Laboratory, Stockholm University, Stockholm, Sweden \\ ${ }^{2}$ Psychology Department, Stockholm University, Stockholm, Sweden \\ ${ }^{3}$ Department of Psychology, Umeå University, Umeå, Sweden \\ ${ }^{4}$ Department of Clinical Sciences, Psychiatry, Umeå University, Umeå, Sweden \\ ${ }^{5}$ Swedish Collegium for Advanced Study, Uppsala, Sweden
}

(Received April 9, 2013; Final Revision December 3, 2013; Accepted December 3, 2013; First Published Online January 22, 2014)

\begin{abstract}
We examined whether conversion to dementia can be predicted by self-reported olfactory impairment and/or by an inability to identify odors. Common forms of dementia involve an impaired sense of smell, and poor olfactory performance predicts cognitive decline among the elderly. We followed a sample of 1529 participants, who were within a normal range of overall cognitive function at baseline, over a 10-year period during which 159 were classified as having a dementia disorder. Dementia conversion was predicted from demographic variables, Mini-Mental State Examination score, and olfactory assessments. Self-reported olfactory impairment emerged as an independent predictor of dementia. After adjusting for effects of other predictors, individuals who rated their olfactory sensitivity as "worse than normal" were more likely to convert to dementia than those who reported normal olfactory sensitivity (odds ratio $[\mathrm{OR}]=2.17$; 95\% confidence interval [CI] [1.40, 3.37]). Additionally, low scores on an odor identification test also predicted conversion to dementia (OR per 1 point increase $=0.89 ; 95 \%$ CI $[0.81,0.98]$ ), but these two effects were additive. We suggest that assessing subjective olfactory complaints might supplement other assessments when evaluating the risk of conversion to dementia. Future studies should investigate which combination of olfactory assessments is most useful in predicting dementia conversion. (JINS, 2014, 20, 209-217)
\end{abstract}

Keywords: Olfaction, Vascular dementia, Alzheimer type dementia, Memory, Smell disorder, Awareness

\section{INTRODUCTION}

Olfactory functions are affected in common forms of dementia disorders, such as Alzheimer's disease and vascular dementia (Gray, Staples, Murren, Dhariwal, \& Bentham, 2001; Mesholam, Moberg, Mahr, \& Doty, 1998). Early in the course of Alzheimer's disease, neurodegeneration (e.g., neuritic plaques, neurofibrillary tangles, or Lewy bodies) occurs in olfactory brain regions such as the olfactory bulb and the anterior olfactory nucleus (Davies, Brooks, \& Lewis, 1993; Ohm \& Braak, 1987). In individuals with mild cognitive impairment, conversion to Alzheimer's disease can be predicted by a poor ability to detect odors (Bacon, Bondi,

Correspondence and reprint requests to: Jonas Olofsson, Psychology Department, Stockholm University, SE-10691 Stockholm, Sweden. E-mail: jonas.olofsson@psychology.su.se
Salmon, \& Murphy, 1998) or identify odors (Devanand et al., 2000). Furthermore, olfactory deficits might precede a decrease in cognitive function among cognitively healthy individuals (Graves et al., 1999; Olofsson et al., 2009, 2010; Swan \& Camelli, 2002; Wilson, Arnold, Tang, \& Benett, 2006). Given that olfactory impairment is present in earlystage dementia, a key question is whether such impairments can be self-assessed by people who are within the normal range of cognitive function, but in a pre-clinical phase of dementia. Awareness of an olfactory impairment and knowledge of its significance could potentially influence an individual's decision to seek medical attention. However, little is known about whether self-assessments of olfactory impairments may be related to pre-clinical dementia. Although only a few studies have investigated self-assessed olfactory impairments among the elderly, they have consistently found a low correlation between self-ratings of 
olfactory ability and performance on standardized tests of olfactory functions. Individuals with a poor ability to detect odors (Nordin, Monsch, \& Murphy, 1995) and identify odors (Murphy et al., 2002; Wehling, Nordin, Espeseth, Reinvang, \& Lundervold, 2011) are often unaware of their deficits. It has even been proposed that olfactory identification deficits, combined with a lack of awareness of these deficits, effectively predicts conversion to dementia in individuals with mild cognitive impairment (Devanand et al., 2000).

Thus, prior studies indicate that subjective reports of olfactory dysfunction might not be useful to indicate olfactory loss in the early stages of dementia. However, no study has investigated the direct relationship between subjective olfactory impairment and later dementia conversion among people who otherwise display a normal level of overall cognitive functioning. It is possible that individuals with intact cognitive abilities might be able to accurately assess a decline in their own olfactory ability, even if these remain within the normal range of function. In individuals where the cause for such subjective olfactory decline is neurodegenerative, olfactory complaints might predict conversion to dementia later in life. Our approach to assess subjective olfactory impairment as a predictor of dementia is motivated by developments in the neuropsychology of memory.

Previously, associations between subjective memory complaints and objective test performance were often reported to be weak or non-existent (Jorm et al., 1994; O'Connor, Pollitt, Roth, Brook, \& Reiss, 1990; O'Hara, Hinrichs, Kohout, Wallace, \& Lemke, 1986; Sunderland, Watts, Baddeley, \& Harris, 1986) and impaired awareness of memory deficits is common, especially in mild cognitive impairment and Alzheimer's disease (Sevush \& Leve, 1993; Vogel et al., 2004). However, longitudinal studies indicate that subjective memory complaints can predict later cognitive decline and conversion to dementia in the general elderly population (Schmand, Jonker, Geerlings, \& Lindeboom, 1997; Schmand, Jonker, Hooijer, \& Lindeboom, 1996; Tobiansky, Blizard, Livingston, \& Mann, 1995; Waldorff, Siersma, Vogel \& Waldemar, 2012). Furthermore, subjective memory complaints have been shown to predict cognitive decline in individuals with normal baseline cognition (Geerlings, Jonker, Bouter, Adèr, \& Schmand, 1999; Wang et al., 2004). Presumably, these individuals are aware of subtle deficits in memory which are not yet severe enough to be reflected in objective tests of memory performance (Geerlings et al., 1999).

On a biological level, subjective memory impairment has been associated with disruptions in the functional integrity of the hippocampal formation, and recent findings suggest that compensatory processes in the frontal lobes might help retain objective memory performance (Erk et al., 2011). Taken together, these findings indicate that subjective memory complaints represent early markers of dementia in the general elderly population, despite the apparently low reliability of subjective memory assessments. This might also be true of complaints about olfactory function. The principal aim of the present study was to investigate the role of subjective olfactory impairment as a potential predictor of later conversion to dementia.
Previous studies have typically identified individuals with olfactory impairments according to a performance cutoff point on a standardized test, and results have shown that these individuals are often unaware of their impairment (Murphy et al., 2002; Wehling et al., 2011). However, there is little consensus on how to define an olfactory impairment, and a discrepancy between olfactory test scores and the subjective experiences of olfactory deficits may be expected. In the present study, we focus instead on the question of whether individuals who classify themselves as having an olfactory impairment have a higher risk of developing dementia later in life compared to those who do not report olfactory dysfunction. We hypothesized that subjective olfactory impairments would complement odor identification test scores in predicting conversion to dementia.

The sample in this study was derived from the Betula study, a large-scale population-based cohort study, and data from over 1500 individuals were included. The sample was screened for general cognitive impairment (Mini-Mental State Examination $[\mathrm{MMSE}]<25$ ), but not for specific cognitive or health impairments to remain representative of the sampled population. Our main analysis addressed whether performance in an olfactory identification test and a self-rating of one's ability to perceive weak odors as "worse than normal" could independently predict conversion to dementia within a 10-year time-span. Follow-up analyses investigated possible effects of attrition, the correlations between subjective and objective olfactory assessments, and determinants of subjective olfactory complaints.

\section{MATERIALS AND METHODS}

\section{Study Population}

The data used in the study were derived from the third wave of data collection in the Betula project, collected in 1998-2000. Betula is a large scale, population-based longitudinal study focused on aging, memory, and health (Nilsson et al., 1997, 2004). In Betula, extensive psychological testing and health assessments are conducted every 5 years. During the third wave of the study, an odor identification test and an assessment of subjective olfactory sensitivity were included for the first time. The study was conducted in Umeå, a city of approximately 100,000 inhabitants located in northern Sweden. The participants were selected based on the city's population registry and participation was voluntary. The Betula study has a narrow age-cohort design in which only participants aged 35, 40, 45 , etc., years old were recruited from the population. The subjects' written consent was obtained in accordance with the Declaration of Helsinki (BMJ 1991; 302: 1194), and the study was approved by the Regional Ethical Vetting Board at Umeå University (approval no. 870303, 97-173, 221/97, 97-173, 03-484, 01-008, 169/02, 02-164, 03-484, 05-082 M, and $08-132 \mathrm{M}$ ). 


\section{Cognitive Measures}

The MMSE was included as a general measure of cognitive function (Folstein, Folstein, \& McHugh, 1975). The MMSE is a brief 30-point questionnaire that assesses general cognitive function and skills in arithmetic, episodic memory, orientation, and language. A test of vocabulary was also included (SRB; Dureman, Kebbon, \& Österberg, 1971) in which 30 low-frequency words are matched to one of five alternatives (synonyms). This test has a format similar to the odor identification task, and was previously used as a non-olfactory control task (Olofsson et al., 2009).

\section{Olfactory Identification and Detection Sensitivity}

Odor identification was assessed using a modified version of the Scandinavian Odor-Identification Test (SOIT), which is a validated and reliable test of odor identification (Nordin, Brämerson, Lidén, \& Bende, 1998). The test consists of 13 different odor stimuli which are considered to represent a wide range of qualities (such as sweet, spicy, woody, citrus, and floral) and are fairly strong in intensity (Nordin et al., 1998). Subjects are provided with a written list of four response choices for each of the presented odors (Bende \& Nordin, 1997), from which they select the best match. The version of the SOIT in the Betula study differed from the original version in that the response alternatives were similar to the corresponding test odorant. This modification made the test relatively difficult in order to avoid ceiling effects.

In the fifth wave of data collection of the Betula study (2008-2010), an odor threshold test was introduced to complement the odor identification test. Odor detection thresholds were determined by presenting participants with pairs of odorous pen-like sticks (Sniffin' Sticks; Hummel, Sekinger, Wolf, Pauli, \& Kobal, 1997) with decreasing concentration levels of $n$-butanol (in total 16 concentrations, where 1 represents the strongest concentration and 16 represents the weakest). The procedure began at an intermediate concentration level (8). One odorous and one odorless stick were presented at each trial. Each stick was presented approximately $2 \mathrm{~cm}$ under the participant's nose every 15-20 s. The participant's task was to report which of the two sticks contained the odor. For those participants who were able to correctly identify the stick that contained the odor four times in a row at the intermediate level, the testing procedure continued with the lowest concentration level (16). When incorrect responses were given at this level, the concentration of the next odor sample was increased by one step without any feedback until the participants gave correct responses at the same concentration level four times in a row. For participants who were unable to discriminate the odorous stick from the blank at the intermediate concentration-level, the odor concentration was increased by one step from the intermediate concentration level until correct responses were given four times in a row. Participants' scores were based on the lowest concentration level they could detect. Thus, a score of 16 reflects high olfactory sensitivity, whereas a score of 1 reflects low sensitivity.

\section{Subjectively Assessed Olfactory Function}

Subjective olfactory status was assessed before the olfactory identification test. The participants were asked the question, "How is your ability to perceive weak odors?" The response alternatives were "no ability," "worse than normal," "normal," and "better than normal." The participants' response distribution indicated that most participants rated their sense of smell as either "normal" $(72.6 \%)$ or "worse than normal" (19.6\%), while no participant reported having "no ability" to perceive weak odors and only $7.8 \%$ perceived their abilities as "better than normal." Because of our focus on olfactory impairment, and because the variable was not suitable for linear statistical models, self-reported olfactory status was collapsed into a dichotomous variable. The response categories "no ability to perceive weak odors" and "worse than normal" were collapsed into a category of olfactory impairment, while the response categories "normal" and "better than normal" were collapsed into a category of normal olfactory function.

\section{Health and Dementia Assessment}

The dementia diagnoses were determined based on a comprehensive review of information obtained from multiple neuropsychological and health-associated assessments, carried out at each test-wave (T1-T5). In addition, an extensive evaluation of medical records to identify symptoms indicative for progressive dementia was conducted at the same occasions throughout the study period. The dementia evaluation process followed a procedure in which participants fulfilling one or several of the following criteria were considered "at a higher risk" and were more extensively evaluated: (a) suspected dementia signs observed by the staff conducting the Betula health assessments and cognitive testing, (b) MMSE performance below 24, (c) a decline in the MMSE score (at least three points) from the previous testing occasion, or (d) a subjective sense of memory impairment reported by the participant. The dementia status of each participant was evaluated by an experienced research psychiatrist, specialist in geriatric and general psychiatry. The diagnosis of Alzheimer's disease was based on the DSM IV criteria (American Psychiatric Association,1994) as well as the core criteria for all-cause dementia and criteria caused by Alzheimer's disease provided by McKhann et al. (2011). The diagnosis of vascular dementia was based on the DSM-IV criteria (American Psychiatric Association, 1994) and on the criteria for possible or probable vascular dementia established by Gorelick et al., (2011). Depressive symptoms were assessed with the Center for Epidemiologic Studies Depression Scale (CES-D; Radloff, 1977).

\section{Participants' Characteristics}

The participants were assessed during the third wave of data collection (1998-2000) and followed for a 10-year time-span until the fifth test wave (2008-2010). During the third wave, 1,924 adult participants (age range: $50-100$ years) with no current diagnosis of dementia were assessed in subjective 
Table 1. Participants' characteristics

\begin{tabular}{|c|c|c|c|}
\hline & $\begin{array}{l}\text { Whole sample } \\
\qquad(N=1529)\end{array}$ & $\begin{array}{l}\text { Dementia conversion } \\
\qquad(N=159)\end{array}$ & $\begin{array}{l}\text { No dementia conversion } \\
\qquad(N=1370)\end{array}$ \\
\hline Age (years), mean $\pm S D$ & $61.2 \pm 11.7$ & $75.5 \pm 7.4$ & $59.5 \pm 10.9$ \\
\hline Sex, female $(\%)$ & $851(55.7)$ & $98(61.6)$ & $753(55)$ \\
\hline Years of education, mean $\pm S D$ & $11.0 \pm 4.1$ & $8.4 \pm 3.1$ & $11.3 \pm 4.1$ \\
\hline MMSE, mean $\pm S D$ & $28.0 \pm 1.4$ & $27.2 \pm 1.4$ & $28.1 \pm 1.4$ \\
\hline Odor identification, mean $\pm S D$ & $7.2 \pm 2.1$ & $5.8 \pm 2.2$ & $7.4 \pm 2.1$ \\
\hline Subjective olfactory complaint (yes) (\%) & $300(19.6)$ & $54(34)$ & $246(18)$ \\
\hline $\begin{array}{l}\text { MMSE decline from third to fifth test wave, } \\
\text { mean } \pm S D(N)\end{array}$ & $0.72 \pm 2.45(790)$ & $6.55 \pm 4.74(33)$ & $0.47 \pm 1.94(757)$ \\
\hline
\end{tabular}

olfactory function, in odor identification, and in the MMSE. Of these 1924 participants, 317 participants had dropped out of the study 10 years later without having received a dementia diagnosis (16\%). These participants had either died $(n=256)$ or quit the study for other reasons $(n=61$; many of these participants had moved away from Umeå) and they were excluded because their dementia status was unknown. Among the remaining 1607 participants, 194 individuals had received a dementia diagnosis by the fifth test wave, while 1413 had not. In the main analysis, participants with baseline MMSE-scores lower than 25 were excluded from our analyses ( $n=78$; of which later dementia conversion, $n=35$ ) because we were mainly interested in participants with no generalized cognitive impairment at baseline. Our final sample thus consisted of 1529 participants, of which 159 had received a dementia diagnosis by the fifth test wave and 1370 had not. Of the dementia cases, $95(59.7 \%)$ were classified as Alzheimer's disease dementia, 53 (33.3\%) as vascular dementia, $3(1.9 \%)$ as Parkinson's disease dementia, $2(1.3 \%)$ as dementia with Lewy Bodies, $1(0.6 \%)$ as frontotemporal dementia, and $5(3.1 \%)$ as unspecified dementia. The participants' characteristics are described in Table 1.

\section{Statistical Analyses}

Logistic regression analysis was performed to investigate the independent effects of olfactory identification and self-ratings on the likelihood of converting to dementia within 10 years. Follow-up analyses were performed with multinomial regression analyses to investigate the predictive utility of subjective olfactory complaints for the two most common dementia subtypes in the sample, Alzheimer's disease dementia and vascular dementia. Follow-up analyses also investigated if vocabulary and depression would absorb variance from the olfactory assessments in the prediction of dementia and possible effects of attrition. To approach a better understanding of the nature of self-reported olfactory impairments, a follow-up logistic regression analysis was conducted to predict the likelihood of subjective olfactory complaints using demographic, cognitive, and olfactory variables. Analyses were two-tailed with an alpha level of 0.05 .

\section{RESULTS}

\section{Subjective Olfactory Complaints and Later Conversion to Dementia}

The main analysis was a logistic regression, with age, gender, years of education, MMSE score, performance in olfactory identification, and subjective olfactory impairment (yes vs. no) as predictor variables, and conversion to dementia within a 10-year time-span as the criterion variable. Results revealed that after adjusting for the demographic variables and MMSE scores, olfactory identification and subjective olfactory impairment significantly and independently predicted conversion to dementia. Individuals who rated their sense of smell as "worse than normal" had 2.17 times the odds of receiving a dementia diagnosis within 10 years compared to those who did not report an olfactory impairment $(p=.001)$, after other predictors were adjusted for. Adding subjective olfactory complaints in the last step of the regression significantly improved the variance explained by the logistic model (omnibus $\chi^{2}=11.61$; $d f=1$; $p=.001$ ) and increased the prediction of dementia from $22 \%$ to $27 \%$. Our analysis revealed further that an increase of 1 point in odor identification performance (range, 0-13) was associated with a decrease in the odds of converting to dementia by a multiplicative factor of $0.89(p<.05)$. Low performance in the MMSE and high age were associated with increased odds of converting to dementia within a 10-year time-span $(p s<.05)$. Years of education and gender had no independent impact on the likelihood of converting to dementia $(p s>.32)$. Table 2 provides coefficients, odds ratios, Wald statistics, and probability values for each of the predictor variables.

\section{Follow-up Analyses}

\section{Predicting dementia subtypes}

The two most common forms of dementia found in our sample were Alzheimer's disease dementia and vascular dementia, and multinomial logistic regression analyses investigated the predictive utility of subjective olfactory complaints and odor identification for these two subtypes 
Table 2. Logistic regression analysis predicting conversion to dementia

\begin{tabular}{lcccr}
\hline \hline Predictor & $B$ & OR & 95\% CI & Wald statistic \\
\hline Age (+1 year) & 0.15 & 1.16 & {$[1.13,1.19]$} & 133.89 \\
Sex (female) & 0.21 & 1.23 & {$[0.82,1.85]$} & 1.01 \\
Years of education (+1 year) & 0.00 & 1.00 & {$[0.95,1.07]$} & 0.02 \\
MMSE (+1 point) & -0.18 & 0.84 & {$[0.73,0.96]$} & .315 \\
Odor identification (+1 point) & -0.11 & 0.89 & {$[0.81,0.98]$} & .892 \\
Subjective olfactory complaint (yes) & 0.77 & 2.17 & {$[1.40,3.37]$} & .013 \\
\hline \hline
\end{tabular}

$\mathrm{CI}=$ confidence interval for odds ratio $(\mathrm{OR})$.

of dementia. The results showed that after adjusting for age, sex, education, and MMSE, olfactory identification significantly predicted conversion to Alzheimer's disease dementia $(p=.03)$, but not to vascular dementia $(p=.51)$. After adjusting for demographic variables, MMSE, and odor identification, subjective olfactory complaints significantly predicted conversion to both Alzheimer's disease dementia $(p<.01)$ and vascular dementia $(p<.05)$.

\section{Semantic memory}

Because odor identification depends to some extent on semantic memory (Hedner, Larsson, Arnold, Zucco, \& Hummel, 2010; Larsson, Nilsson, Olofsson, \& Nordin, 2004; Olofsson, Rogalski, Harrison, Mesulam, \& Gottfried, 2013; Schab, 1991), the logistic regression analysis was repeated to include vocabulary. The results showed that the association between the vocabulary test SRB and dementia was not significant $(p=.30)$, and both odor identification and subjective olfactory impairment were unchanged as significant predictors of dementia conversion after including SRB $(p \mathrm{~s}<.05)$.

\section{Depression}

Both olfactory dysfunction (Pause, Miranda, Göder, Aldenhoff, \& Ferstl, 2001) and a higher risk for developing dementia (for a review, see Da Silva, Gonçalves-Pereira, Xavier, \& Mukaetova-Ladinska, 2013) have previously been associated with depression. Furthermore, depression is linked to memory complaints in the elderly (Jorm, Christensen, Korten, Jacomb, \& Henderson, 2001). In a similar manner, depressive symptomatology might be related to subjective olfactory complaints. Thus, we addressed the possibility that the relationship between subjective olfactory impairment and dementia might be due to shared variance with depressive symptoms by including an assessment of depressive symptomatology. However, CES-D scores were not a significant predictor of dementia $(p=.633)$ and the relationships between the two olfactory assessments and dementia remained significant $(p \mathrm{~s}<.05)$.

\section{Attrition}

Attrition is a common problem in longitudinal research on cognitive aging, as participants who remain in the study tend to perform better in cognitive tasks than those dropping out (e.g., Cooney, Schaie, \& Willis, 1988). In this study, we excluded participants without a dementia diagnosis who cancelled their participation, as their dementia status became unknown. As noted above, these excluded participants were divided into two groups: "deceased" and "other." Follow-up analyses compared these groups to a reference group of all included participants, both those who had received a dementia diagnosis and those who had not. The "other" group did not differ from the reference group on either odor identification $(\mathrm{t}=0.807 ; d f=1,666 ; p=.42)$ or subjective olfactory function $\left(\mathrm{U}=46,454,500 ; N_{1}=61 ; N_{2}=1607\right.$; $p=.32$ ).

In contrast, the "deceased" group had significantly lower scores in olfactory identification $(t=8.786 ; d f=1,861$; $p<.001)$ and were more likely to rate their sense of smell as impaired $\left(U=180,897 ; N_{1}=256 ; N_{2}=1607 ; p<.001\right)$ than participants in the reference group. Importantly, however, a follow-up logistic regression analysis revealed that the relationship between olfactory assessments and mortality became statistically insignificant $(p s>.13)$ after controlling for age, gender, years of education, and cognitive ability (MMSE) at baseline. Thus, the association between olfactory impairment and attrition was fully accounted for by the fact that the "deceased" group was, on average, older $(t=18.516 ; d f=1,861 ; p<.001)$, less educated $(t=9.037$; $d f=1,861 ; p<.001)$, performed worse in the MMSE $(t=8.581 ; d f=1,892 ; p<.001)$, and had a higher frequency of males $\left(U=185,309 ; N_{1}=256 ; N_{2}=1,607 ; p<.05\right)$ than those who remained in the study. Since demographic (age, gender, years of education) and cognitive (MMSE, SRB) variables were taken into account in the main analysis, effects of attrition were unlikely to influence the observed relationship between olfactory assessments and conversion to dementia.

Furthermore, it should be noted that a higher number of individuals had died without a dementia diagnosis $(n=256)$ compared to those who had received a diagnosis at the end of the follow-up period $(n=159)$. Thus, the lack of direct relationship between olfactory assessments and attrition could not be attributed to lower statistical power in this analysis. We conclude that attrition was unlikely to affect the main findings that objective and subjective olfactory deficits independently predicted the likelihood of dementia conversion within 10 years. 
Table 3. Participant's characteristics from the fifth wave (2008-2010)

Age (years), mean $\pm S D$

Sex, female $(\%)$

Years of education, mean $\pm S D$

MMSE, mean $\pm S D$

Odor identification, mean $\pm S D$

Odor threshold, mean $\pm S D$

Subjective olfactory complaint (yes) $(\%)$

$61.5 \pm 14.2$

$521(53.4)$

$12.72 \pm 4.1$

$28.2 \pm 1.4$

$6.9 \pm 2.2$

$5.7 \pm 3.0$

$218(22.3)$

\section{Determinants and aspects of subjective olfactory} assessment

Follow-up logistic regression analysis was conducted to predict the likelihood of reporting an olfactory impairment using demographic, cognitive, and olfactory variables. Data from participants in the fifth wave of data collection were included if the participants if they had no diagnosed dementia disorder, an MMSE score of 25 or above, and had completed subjective olfactory assessments, as well as odor identification and odor threshold assessments. This resulted in 976 participants eligible for follow-up analysis. The characteristics of these individuals are described in Table 3.

Age, sex, years of education, MMSE, odor thresholds, and odor identification were entered as predictor variables and subjective olfactory complaint was the criterion measure. Odds ratios of reporting an olfactory impairment was significantly increased by higher age $(p<.05)$, while female sex was associated with lower likelihood of subjective olfactory complaints $(p<.01)$. Years of education $(p=.48)$ and MMSE $(p=.22)$ were not significant as predictors for subjective olfactory impairment. Self-rated olfaction was independently predicted by both odor identification and odor thresholds ( $p s<.02$ ), with decreasing olfactory performance associated with increased odds of reporting an olfactory impairment. Although the olfactory self-assessment questionnaire item referred specifically to the "ability to perceive weak odors," odor identification emerged as a significant predictor, in addition to olfactory thresholds. Thus, when asked about their ability to detect weak odors, participants' responses reflect both sensory (detection) and cognitive (identification) olfactory abilities.

The full model significantly predicted reports of an olfactory impairment (omnibus $\chi^{2}=50.92, .97 ; d f=6 ; p<.001$ ).

However, calculations of Cox \& Snell R Square and Nagelkerke R Square showed that the model only accounted for between $5.1 \%$ and $7.8 \%$ of the variance in self-assessed olfactory impairment. Calculations of Cox \& Snell R Square and Nagelkerke R Square revealed further that adding odor thresholds and odor identification to the model increased explained variance by only $2.0 \%$ to $2.9 \%$, indicating relatively low distinguishing ability for olfactory tests in this sample. The results of the logistic regression analysis are summarized in Table 4.

\section{DISCUSSION}

Subjective assessments of olfactory function were previously viewed as poor indicators of actual olfactory loss. As a consequence, subjective olfactory complaints could not reflect the olfactory impairments that are characteristic of early-stage dementia syndromes (Devanand et al., 2000; Larsson et al., 1999). However, no previous study has investigated the predictive role of self-reported olfactory impairment on dementia conversion in cognitively healthy individuals. This study showed that rating one's sense of smell as "worse than normal" independently predicted dementia conversion within a 10-year time-span in adults without generalized cognitive impairment at baseline. The effect was present in both major dementia subtypes, Alzheimer's disease dementia and vascular dementia, and remained stable after controlling for demographic variables, cognitive ability, odor identification ability, and symptoms of depression. Thus, our results provide the first evidence for an independent role of subjective olfactory complaints in the early detection of dementia.

The results of this study aligns with findings that subjective memory complaints can predict later dementia conversion (e.g., Geerlings et al., 1999; Schmand et al., 1996, 1997), despite that self-assessments of memory function often appear unreliable in cross-sectional studies (e.g., Jorm et al., 1994; O'Connor et al., 1990). Similarly, the cross-sectional follow-up analyses conducted in this study suggest that olfactory ratings are only weakly related to the distribution of scores on olfactory tests, which is in agreement with previous research (Murphy et al., 2002; Shu et al., 2009; Wehling et al., 2011). A possible explanation for this apparent unreliability of olfactory self-evaluations might be that individuals

Table 4. Logistic regression analysis of determinants of subjective olfactory complaints

\begin{tabular}{lcccr}
\hline \hline Predictor & $B$ & $O R$ & $95 \%$ CI & Wald statistic \\
\hline Age (+1 year) & 0.02 & 1.02 & {$[1.00,1.03]$} & 5.39 \\
Sex (female) & -0.52 & 0.59 & {$[0.43,0.81]$} & .02 \\
Years of education (+1 year) & 0.02 & 1.02 & {$[0.97,1.06]$} & .001 \\
MMSE (+1 point) & 0.08 & 1.08 & {$[0.96,1.22]$} & .72 \\
Odor thresholds (+1 point) & -0.10 & 0.91 & {$[0.86,0.96]$} & .50 \\
Odor identification(+1 point) & -0.10 & 0.91 & {$[0.84,0.98]$} & .52 \\
\hline \hline
\end{tabular}

$\mathrm{CI}=$ confidence interval for odds ratio (OR). 
are often unaware of their olfactory abilities compared to those of other people. For example, when assessing one's own olfactory ability, a cognitively healthy respondent may compare his or her present level of olfactory sensitivity to past levels of sensitivity rather than comparing it to other people's sensitivity.

Importantly, from the perspective of identifying the risk of future dementia conversion, this intra-individual change may actually be of higher diagnostic utility than betweenindividual comparisons, especially given the large individual differences in baseline olfactory sensitivity (Stevens, Cain, \& Burke, 1988). A low correlation between subjective and objective sensitivity may therefore not impair the utility of subjective olfactory assessments in this context. In future assessments of subjective olfactory decline, we propose that refined scales measuring self-reported olfactory function might focus on intra-individual changes in olfactory abilities (Bahar-Fuchs, Moss, Rowe, \& Savage, 2011; Djordjevic, Jones-Gotman, De Sousa, \& Chertkow, 2008) rather than comparisons to other individuals. Moreover, although the question of one's ability to perceive weak odors was formulated to address detection sensitivity, our results suggest that participants' responses were also influenced by cognitive olfactory abilities. It is yet unclear to what extent sensory and cognitive olfactory functions influence the subjective evaluation in different participants. Further research is needed to investigate these metacognitive aspects of olfaction in the context of dementia.

We found that performance in olfactory identification could independently predict conversion to dementia within a 10-year time-span. The result supports previous research (Graves et al., 1999; Olofsson et al., 2009, 2010; Swan \& Camelli, 2002; Wilson et al., 2006) and demonstrates the importance of olfactory assessments for the early detection of dementia. The present results suggest that subjective reports might complement olfactory tests, but further research is needed to establish the optimal combination of subjective and objective olfactory assessments to predict later conversion to dementia.

Olfactory self-evaluation might have limited utility in certain clinical settings, as individuals with cognitive impairment might not be aware of their olfactory loss. While we excluded participants with generalized cognitive impairment at baseline, it is possible that the outcome would have differed if our sample had contained more individuals with cognitive impairment but no dementia, as these individuals might have a lack of insight into olfactory deficits, coupled with an increased risk of later dementia conversion (Devanand et al., 2000). Furthermore, our screening procedure did not involve health variables other than dementia diagnosis. While this makes the sample more similar to the general population, it also means including participants with, for example, cardiovascular problems, which might score normally on the MMSE but are nevertheless at increased risk of dementia, in particular the vascular type.

However, subjective olfactory performance could predict conversion to Alzheimer's disease as well as vascular dementia in our sample, and given that the Betula study design includes a neuropsychiatric evaluation of participants showing signs of dementia, it seems unlikely that patients with undiagnosed vascular dementia but no MMSE impairment would constitute a significant proportion of the baseline sample. In light of the present findings, we emphasize a multi-factorial approach in predicting and evaluating dementia. Information about olfactory status, irrespective of whether the information is based on self-reports or objective testing, could be viewed as a supplement to conventional dementia investigation. However, we note that the specificity obtained with the present procedure was rather low, since approximately five of six people who reported a poor ability to perceive weak odors did not convert to dementia.

There are many possible etiologies other than dementia for poor olfactory function (e.g., Nordin \& Brämerson, 2008), indicating that attempts should be made to rule out causes other than dementia in cases of poor olfactory function. In conclusion, the results of this study support earlier findings suggesting that olfactory impairment is a pre-clinical marker for dementia (Albers, Tabert, \& Devanand, 2006; Morgan, Nordin, \& Murphy, 1995; Olofsson et al., 2009; Ponsen et al., 2004; Schubert et al., 2008). Most importantly, the results reveal that subjective olfactory impairment constitutes an early marker of an impending dementia. Subjective olfactory evaluations might thus become a supplementary tool in the early detection of dementia.

\section{CONFLICT OF INTEREST}

The authors declare no conflict of interest.

\section{ACKNOWLEDGMENTS}

The Betula Study is funded by the Swedish Research Council (345-2003-3883, 315-2004-6977). This study was further supported by grants from the Swedish Research Council to Jonas Olofsson (421-2012-806) and Maria Larsson (421-2011-1792). The authors thank Maria Josefsson and Annelie Nordin at Umeå University for valuable consultation and assistance, and Caitlin B. Hawley for proofreading of the manuscript.

\section{REFERENCES}

Albers, M.W., Tabert, M.H., \& Devanand, D.P. (2006). Olfactory dysfunction as a predictor of neurodegenerative disease. Current Neurology and Neuroscience Reports, 6(5), 379-386. doi:10.1007/s11910-996-0018-7

American Psychiatric Association. (1994). Diagnostic and Statistical Manual of Mental Disorders (4th ed.). Washington, DC: American Psychiatric Association Press.

Bacon, A.W., Bondi, M.W., Salmon, D.P., \& Murphy, C.M. (1998). Very early changes in olfactory functioning due to Alzheimer's disease and the role of apolipoprotein E in olfaction. Annuals of the New York Acadamey of Sciences, 855, 723-731. doi:10.1111/ j.1749-6632.1998.tb10651.x

Bahar-Fuchs, A., Moss, S., Rowe, C., \& Savage, G. (2011). Awareness of olfactory deficits in healthy aging, amnestic mild cognitive impairment and Alzheimer's disease. International 
Psychogeriatrics, 23(7), 1097-1106. doi:10.1017/S10416102100 02371

Bende, M., \& Nordin, S. (1997). Perceptual learning in olfaction: Professional wine tasters versus controls. Physiology \& Behavior, 62(5), 1065-1070. doi:10.1016/S0031-9384(97)00251-5

Cooney, T.M., Schaie, W., \& Willis, S.L. (1988). The relationship between prior functioning on cognitive and personality dimensions and subject attrition in longitudinal research. Journal of Geronology, 43, 1217.

Da Silva, J., Gonçalves-Pereira, M., Xavier, M., \& MukaetovaLadinska, E.B. (2013). Affective disorders and risk of developing dementia: Systematic review. The British Journal ofPpsychiatry: The journal of Mental Science, 202, 177-186. doi:10.1192/ bjp.bp.111.101931

Davies, D.C., Brooks, J.W., \& Lewis, D.A. (1993). Axonal loss from the olfactory tracts in Alzheimer's disease. Neurobiology of Aging, 14, 353-357. doi:10.1016/0197-4580(93)90121-Q

Devanand, D., Michaels-Marston, K.S., Xinhua, L., Pelton, G.H., Padilla, M., Marder, K., \& Mayeux, R. (2000). Olfactory deficits in patients with mild cognitive impairment predict Alzheimer's disease at follow-up. American Journal of Psychiatry, 157, 1399-1405. doi:10.1176/appi.ajp.157.9.1399

Djordjevic, J., Jones-Gotman, M., De Sousa, K., \& Chertkow, H. (2008). Olfaction in patients with mild cognitive impairment and Alzheimer's disease. Neurobiology of Aging, 29(5), 693-706. doi:10.1016/j.neurobiolaging.2006.11.014

Dureman, I., Kebbon, L., \& Österberg, E. (1971). A manual to the DS-battery. Psykologiförbundet AB, Stockholm.

Erk, S., Spottke, A., Meisen, A., Wagner, M., Walter, H., \& Jessen, F. (2011). Evidence of neuronal compensation during episodic memory in subjective memory impairment. Archives of General Psychiatry, 68(8), 845-852. doi:10.1001/archgenpsychiatry. 2011.80

Folstein, M.F., Folstein, S.E., \& McHugh, P.R. (1975). Mini-mental state: A practical method for grading the cognitive state of patients for the clinician. Journal of Psychiatric Research, 12, 189-198.

Geerlings, M.I., Jonker, C., Bouter, L.M., Adèr, H.J., \& Schmand, B. (1999). Association between memory complaints and incident Alzheimer's disease in elderly people with normal baseline cognition. The American Journal of Psychiatry, 156(4), 531-537.

Gorelick, P.B., Scuteri, A., Black, S.E., DeCarli, C., Greenberg, S.M., Iadecola, C., ... Seshadri, S. (2011). Vascular contributions to cognitive impairment and dementia: A statement for healthcare professionals from the American heart association/American stroke association. Stroke, 42(9), 2672-2713. doi:10.1161/ STR.0b013e3182299496

Graves, A.B., Bowen, J.D., Rajaram, L., McCormick, W.C., McCurry, S.M., Schellenberg, G.D., \& Larson, E.B. (1999). Impaired olfaction as a marker for cognitive decline: Interaction with apolipoprotein E \&4 status. Neurology, 53(7), 1480-1487. doi:10.1212/WNL.53.7.1480

Gray, A.J., Staples, V., Murren, K., Dhariwal, A., \& Bentham, P. (2001). Olfactory identification is impaired in clinic-based patients with vascular dementia and senile dementia of Alzheimer type. International Journal of Geriatric Psychiatry, 16(5), 513-517. doi:10.1002/gps.383

Hedner, M., Larsson, M., Arnold, N., Zucco, G.M., \& Hummel, T. (2010). Cognitive factors in odor detection, odor discrimination, and odor identification tasks. Journal of Clinical and Experimental Neuropsychology, 32(10), 1062-1067. doi:10.1080/138033910036 83070
Hummel, T., Sekinger, B., Wolf, S.R., Pauli, E., \& Kobal, G. (1997). 'Sniffin' sticks': Olfactory performance assessed by the combined testing of odor identification, odor discrimination and olfactory threshold. Chemical Senses, 22, 39-52. doi:10.1586/ ern.09.115

Jorm, A.F., Christensen, H., Henderson, A.S., Korten, A.E., Mackinnon, A.J., \& Scott, R. (1994). Complaints of cognitive decline in the elderly: A comparison of reports by subjects and informants in a community survey. Psychological Medicine, 24, 365-374. doi:10.1017/S0033291700027343

Jorm, A.F., Christensen, H., Korten, A.E., Jacomb, P.A., \& Henderson, A.S. (2001). Memory complaints as a precursor of memory impairment in older people: A longitudinal analysis over 7-8 years. Psychological Medicine, 31, 441-449.

Larsson, M., Nilsson, L.G., Olofsson, J.K., \& Nordin, S. (2004). Demographic and cognitive predictors of cued odor identification: Evidence from a population-based study. Chemical Senses, 29(6), 547-554. doi:10.1093/chemse/bjh059

Larsson, M., Semb, H., Winblad, B., Amberla, K., Wahlund, L.-O., \& Bäckman, L. (1999). Odor identification in normal aging and early Alzheimer's disease: Effects of retrieval support. Neuropsychology, 13, 1-7. doi:10.1037/0894-4105.13.1.47

McKhann, G.M., Knopman, D.S., Chertkow, H., Hyman, B.T., Jack, C.R., Kawas, C.H., ... Phelps, C.H. (2011). The diagnosis of dementia due to Alzheimer's disease: Recommendations from the National Institute on Aging-Alzheimer's Association workgroups on diagnostic guidelines for Alzheimer's disease. Alzheimers and Dementia, 7(3), 263-269. doi:10.1016/j.jalz. 2011.03.005

Mesholam, R.I., Moberg, P.J., Mahr, R.N., \& Doty, R.L. (1998). Olfaction in neurodegenerative disease: A meta-analysis of olfactory functioning in Alzheimer's and Parkinson's diseases. Archives of Neurology, 55(1), 84-90. doi:10.1001/archneur. 55.1 .84

Morgan, C.D., Nordin, S., \& Murphy, C. (1995). Odor identification as an early marker for Alzheimer's disease: Impact of lexical functioning and detection sensitivity. Journal of Clinical and Experimental Neuropsychology, 17(5), 793-803. doi:10.1080/ 01688639508405168

Murphy, C., Schubert, C.R., Cruickshanks, K.J., Klein, B.E., Klein, R., \& Nondahl, D.M. (2002). Prevalence of olfactory impairment in older adults. The Journal of the American Medical Association, 288(18), 2307-2312. doi:10.1001/jama.288.18. 2307

Nilsson, L.G., Adolfsson, R., Backman, L., de Frias, C.M., Molander, B., \& Nyberg, L. (2004). Betula: A prospective cohort study on memory, health and aging. Aging Neuropsychology and Cognition, 11, 134-148. doi:10.1080/13825580490511026

Nilsson, L.G., Backman, L., Erngrud, K., Nyberg, L., Adolfsson, R., Bucht, G., \& Winblad, B. (1997). The Betula prospective cohort study: Memory, health and aging. Aging Neuropsychology and Cognition, 4, 1-32. doi:10.1080/13825589708256633

Nordin, S., \& Brämerson, A. (2008). Complaints of olfactory disorders: Epidemiology, assessment and clinical implications. Current Opinion in Allergy and Clinical Immunology, 8, 10-15. doi:10.1097/ACI.0b013e3282f3f473

Nordin, S., Brämerson, A., Lidén, E., \& Bende, M. (1998). The Scandinavian odor-identification test: Development, reliability, validity and normative data. Acta Oto-Laryngologica, 118, 226-234. doi:10.1080/00016489850154946

Nordin, S., Monsch, A., \& Murphy, C. (1995). Unawareness of smell loss in normal aging and Alzheimer's disease: Discrepancy 
between self-reported and diagnosed smell sensitivity. Journal of Gerontology, 50(B), 187-192. doi:10.1093/geronb/50B.4.P187

O'Connor, D.W., Pollitt, P.A., Roth, M., Brook, P.B., \& Reiss, B.B. (1990). Memory complaints and impairment in normal, depressed, and demented elderly persons identified in a community survey. Archives of General Psychiatry, 47, 224-227. doi:10.1001/ archpsyc.1990.01810150024005

O’Hara, M.W., Hinrichs, J.V., Kohout, F.J., Wallace, R.B., \& Lemke, J.H. (1986). Memory complaint and memory performance in the depressed elderly. Psychology and Aging, 1, 208-214. doi:10.1037/0882-7974.1.3.208

Ohm, T.G., \& Braak, H. (1987). Olfactory bulb changes in Alzheimer's disease. Acta Neuropathologica (Berlin), 73, 365-369. doi:10.1007\%2FBF00688261

Olofsson, J.K., Nordin, S., Wiens, S., Hedner, M., Nilsson, L.-G., \& Larsson, M. (2010). Odor identification impairment in elderly ApoE-e4 carriers is independent of dementia. Neurobiology of Aging, 31, 567-577. doi:10.1016/j.neurobiolaging.2008.05.019

Olofsson, J.K., Rogalski, E., Harrison, T., Mesulam, M.-M., \& Gottfried, J. (2013). A cortical pathway to odor naming: Evidence from primary progressive aphasia. Brain, 136, 1245-1259. doi:10.1093/brain/awt019

Olofsson, J.K., Rönnlund, M., Nordin, S., Nyberg, L., Nilsson, L.-G., \& Larsson, M. (2009). Odor identification deficit as a predictor of five-year global cognitive change: Interactive effects with age and ApoE-epsilon4. Behavior Genetics, 39, 496-503. doi:10.1007/ s10519-009-9289-5

Pause, B.M., Miranda, A., Göder, R., Aldenhoff, J.B., \& Ferstl, R. (2001). Reduced olfactory performance in patients with major depression. Journal of Psychiatric Research, 35, 271-277. doi:10.1016/S0022-3956(01)00029-2

Ponsen, M.M., Stoffers, D., Booij, J., van Eck-Smit, B.L.F., Wolters, E.Ch., \& Berendse, H.W. (2004). Idiopathic hyposmia as a preclinical sign of Parkinson's disease. Annuals of Neurology, 56, 173-181. doi:10.1002/ana.20160

Radloff, L.S. (1977). The CES-D Scale: A self-report depression scale of research in the general population. Applied Psychological Measurement, 1, 385-401. doi:10.1177/014662167700100306

Schab, F.R. (1991). Odor memory: Taking stock. Psychological Bulletin, 2, 242-251. doi:10.1037\%2F\%2F0033-2909.109.2.242

Schmand, B., Jonker, C., Geerlings, M.I., \& Lindeboom, J. (1997). Subjective memory complaints in the elderly: Depressive symptoms and future dementia. British Journal of Psychiatry, 171, 373-376. doi:10.1212/WNL.46.1.121

Schmand, B., Jonker, C., Hooijer, C., \& Lindeboom, J. (1996). Subjective memory complaints may announce dementia. Neurology, 46, 121-125. doi:10.1212/WNL.46.1.121

Schubert, C.R., Carmichael, L.L., Murphy, C., Klein, B.E.K., Klein, R., \& Cruickshanks, K.J. (2008). Olfaction and the 5-year incidence of cognitive impairment in an epidemiological study of older adults. Journal of the American Geriatrics Society, 56, 1517-1521. doi:10.1111/j.1532-5415.2008.01826.x

Sevush, S., \& Leve, N. (1993). Denial of memory deficit in Alzheimer's disease. American Journal of Psychiatry, 150, $748-751$.

Shu, C.-H., Hummel, T., Lee, P.-L., Chiu, C.-H., Lin, S.-H., \& Yuan, B.-C. (2009). The proportion of self-rated olfactory dysfunction does not change across the life span. American Journal of Rhinology \& Allergy, 23(4), 413-416. doi:10.2500/ ajra.2009.23.3343

Stevens, J., Cain, W., \& Burke, R. (1988). Variability of olfactory thresholds. Chemical Senses, 13(4), 643-653. doi:10.1093/ chemse/13.4.643

Sunderland, A., Watts, K., Baddeley, A.D., \& Harris, J.E. (1986) Subjective memory assessment and test performance in elderly adults. Journal of Gerontology, 41, 376-384. doi:10.1093\% 2Fgeronj\%2F41.3.376

Swan, G.E., \& Carmelli, D. (2002). Impaired olfaction predicts cognitive decline in nondemented older adults. Neuroepidemiology, 21, 58-67. doi:10.1159/000048618

Tobiansky, R., Blizard, R., Livingston, G., \& Mann, A. (1995). The Gospel Oak Study stage IV: The clinical relevance of subjective memory impairment in older people. Psychological Medicine, 25, 779-786. doi:10.1017\%2FS00332917000 35029

Vogel, A., Stokholm, J., Gade, A., Andersen, B.B., Hejl, A.-M., \& Waldemar, G. (2004). Awareness of deficits in mild cognitive impairment and Alzheimer's disease: Do MCI patients have impaired insight? Dementia and Geriatric Cognitive Disorders, 17(3), 181-187. doi :10.1159\%2F000076354

Waldorff, F.B., Siersma, V., Vogel, A., \& Waldemar, G. (2012). Subjective memory complaints in general practice predicts future dementia: A 4-year follow-up study. International Journal of Geriatric Psychiatry, 27(11), 1180-1188. doi:10.1002\%2Fgps.3765

Wang, L., Van Belle, G., Crane, P.K., Kukull, W.A., Bowen, J.D., McCormick, W.C., \& Larson, E.B. (2004). Subjective memory deterioration and future dementia in people aged 65 and older. Journal of the American Geriatrics Society, 52(12), 2045-2051. doi:10.1111/j.1532-5415.2004.52568.x

Wehling, E., Nordin, S., Espeseth, T., Reinvang, I., \& Lundervold, A. (2011). Unawareness of olfactory dysfunction and its association with cognitive functioning in middle aged and old adults. Archives of Clinical Neuropsychology, 26, 260-269. doi:10.1093\%2Farclin\%2Facr019

Wilson, R.S., Arnold, S.E., Tang, X., \& Bennett, D.A. (2006). Odor identification and decline in different cognitive domains in old age. Neuroepidemiology, 26, 61-67. doi:10.1159\%2F0000 90250 